\title{
Filigrane
}

Écoutes psychothérapiques

\section{Interprétation et interprétariat : chassé-croisé en thérapies analytiques plurilingues}

\author{
Isabelle Boivin, Camille Brisset et Yvan Leanza
}

Volume 20, numéro 2, automne 2011

URI : https://id.erudit.org/iderudit/1007614ar

DOI : https://doi.org/10.7202/1007614ar

Aller au sommaire du numéro

Éditeur(s)

Revue Santé mentale au Québec

ISSN

1192-1412 (imprimé)

1911-4656 (numérique)

Découvrir la revue

Citer cet article

Boivin, I., Brisset, C. \& Leanza, Y. (2011). Interprétation et interprétariat : chassé-croisé en thérapies analytiques plurilingues. Filigrane, 20(2), 107-122. https://doi.org/10.7202/1007614ar

\section{Résumé de l'article}

Cet article porte sur l'interprétation, processus au coeur de l'activité aussi bien de l'interprète communautaire que du thérapeute d'approche analytique. Chacun agit selon une démarche particulière, sur une base de référents théoriques et techniques propres à sa profession ou à sa fonction; et tous deux reçoivent un ensemble d'informations qu'ils doivent comprendre, transformer et transmettre à nouveau. Cependant, ni l'un ni l'autre ne se positionne comme simple passeur d'information, mais bien comme interlocuteur à part entière dans un dialogue à trois, participant à la construction d'une

intercompréhension. Les auteurs passent en revue des notions clés de la thérapie analytique, comme la neutralité, le transfert et le contre-transfert, qui doivent être repensées dans le cas où un interprète communautaire est nécessaire pour établir le dialogue. Il apparaît qu'interprète et thérapeute doivent se questionner sur la place à accorder à la neutralité et à leur propre subjectivité dans la communication, en fonction des objectifs thérapeutiques. 


\section{Interprétation et interprétariat: chassé- croisé en thérapies analytiques plurilingues}

\section{Isabelle Boivin, Camille Brisset et Yvan Leanza}

Cet article porte sur l'interprétation, processus au cœur de l'activité aussi bien de l'interprète communautaire que du thérapeute $d$ 'approche analytique. Chacun agit selon une démarche particulière, sur une base de référents théoriques et techniques propres à sa profession ou à sa fonction; et tous deux reçoivent un ensemble d'informations qu'ils doivent comprendre, transformer et transmettre à nouveau. Cependant, ni I'un ni l'autre ne se positionne comme simple passeur d'information, mais bien comme interlocuteur à part entière dans un dialogue à trois, participant à la construction d'une intercompréhension. Les auteurs passent en revue des notions clés de la thérapie analytique, comme la neutralité, le transfert et le contre-transfert, qui doivent être repensées dans le cas où un interprète communautaire est nécessaire pour établir le dialogue. II apparaît qu'interprète et thérapeute doivent se questionner sur la place à accorder à la neutralité et à leur propre subjectivité dans la communication, en fonction des objectifs thérapeutiques.

$E^{n}$ n santé mentale, peut-être plus que dans les autres domaines de la santé, le dialogue entre le patient et le praticien est crucial. Il est la base à partir de laquelle l'évaluation du patient et son traitement sont élaborés. La langue est le principal moyen par lequel les patients transmettent leur monde vécu. Elle est aussi le moyen par lequel les praticiens partagent leur compréhension de la situation de leurs patients. Il est communément admis que la dynamique thérapeutique analytique ne peut se mettre en place sans échange verbal, l'objectif principal étant de rendre conscient un phénomène psychique inconscient et donc de mettre en mot ce qui ne l'a pas encore été. Cet objectif et le cadre de la thérapie analytique, qui inclut une compréhension du langage non-verbal et des processus projectifs, présupposent que le patient et le thérapeute partagent un même code symbolique permettant le dialogue, en d'autres termes qu'ils parlent la même langue. Se pose alors la question de la possibilité d'un dialogue lorsque ce code est absent. 
Le recours à un interprète communautaire est une solution de plus en plus courante, mais induit de fait une modification dans la dynamique thérapeutique. Il n'y a plus deux acteurs en présence, mais bien trois. Les différents éléments du processus thérapeutique, tels que l'interprétation, la neutralité, les transferts et contre-transferts et les résistances s'y retrouvent, mais se manifestent différemment. Le cadre, qui permet de maintenir les éléments du contexte constants afin de rendre possible l'exploration des processus qui s'y jouent (Bleger, 1979, 255-256), est aussi mis au défi, ne serait-ce que par les rôles que l'interprète endossera dans la dynamique à trois.

Cet article explore l'influence de l'interprète communautaire sur la communication dans un cadre thérapeutique, ainsi que les différents moyens de l'y inclure. Il convient ici de situer le lieu à partir duquel nous prenons position. En effet, notre expertise, développée dans le cadre des activités du laboratoire Psychologie et Cultures (École de Psychologie, Université Laval), se situe davantage au niveau de l'interprétariat communautaire que de celui de l'approche psychanalytique. Toutefois, deux des auteurs, IB et YL, possèdent une formation clinique incluant l'approche psychodynamique.

\section{Définitions et description}

Donnons tout d'abord une définition: «Dans le sens le plus général, l’interprétariat communautaire désigne l'activité d'interprétation [linguistique] en milieu institutionnel d'une société donnée dans lequel les prestataires de service public et les clients ne parlent pas la même langue» (Pöchhacker, 1999, 126, traduction libre). Les actions de l'interprète visent à rendre la communication possible entre un usager de services et un praticien qui ne partagent pas le même langage.

Nombre de caractéristiques de l'interprète communautaire le distinguent de l'interprète de conférence, qui reste la référence canonique en matière d'interprétariat. Le premier est partie prenante de l'interaction, contrairement au second qui se situe à l'extérieur de celle-ci. Pour cette raison, l'interprète communautaire est parfois nommé interprète de dialogues (p. ex. Wadensjö, 2002) ou interprète en milieu social. De ce fait, il n'a pas accès au texte avant la rencontre. Il réalise généralement une traduction consécutive, aussi nommée par paragraphe, qui se différencie de la traduction simultanée demandée et attendue lors des conférences. Son niveau de formation en interprétariat peut varier grandement, de rien du tout à plusieurs années de formation universitaire. Un praticien peut ainsi se retrouver en présence d'interprètes informels, c'està-dire sans formation, ou professionnels. Dans la majorité des cas, l'interprète 
appartient à la même communauté que les personnes pour lesquelles il interprète, d'où la désignation de communautaire. Il peut également intervenir dans diverses institutions, et donc suivre un même usager dans son parcours institutionnel, ou avoir un poste fixe au sein d'un même établissement. Tous ces éléments présentent des implications sur l'alliance de travail, qui est alors plus ou moins développée et négociée à travers le temps, entre l'interprète, les professionnels de la santé et les institutions.

\section{Interpréter et interpréter}

Les thérapies analytiques ont pour procédé principal l'interprétation. Tous les autres procédés techniques y sont subordonnés, tant au niveau pratique que théorique (Greenson, 1977, 53). L'interprétation consiste à rendre conscient un fait psychique inconscient ou préconscient. Le mode d'écoute du thérapeute est l'écoute flottante, qui consiste à ne rien privilégier par avance de ce que dit le patient, de sorte que les associations puissent émerger d'ellesmêmes. Greenson (1968, 124-125) décrit ce mode d'écoute en trois étapes. Le thérapeute traduit d'abord les productions du patient, telles que ses pensées, ses fantasmes, ses sentiments, son comportement et ses pulsions, dans leur langue inconsciente d'origine. Il synthétise ensuite les éléments inconscients dans des insights significatifs pour les communiquer au patient. Le thérapeute est donc «celui qui comprend et qui véhicule les insights» (Greenson, 1968, 125). Or, ce processus d'écoute, de compréhension et de synthèse n'est pas sans rappeler le processus réalisé par l'interprète lui-même. L'interprète doit forcément interpréter pour rendre le discours intelligible, il ne fait pas que traduire. Alors que la traduction correspond à l'échange de la signification dénotative d'un mot, d'une expression ou d'une phrase dans une langue vers une autre, l'interprétation met l'accent sur l'échange du sens connotatif entre les langues afin que soient véhiculés affects et sens (Westermeyer, 1993, 129). Pour remplir sa fonction de passeur, l'interprète réalise un ensemble de tâches complexes. Il doit d'abord recevoir un ensemble d'informations, en dégager un sens, trouver un équivalent dans l'autre langue et transmettre ce sens. Son écoute doit être d'une certaine façon flottante et privilégier le sens et non la traduction littérale, mot pour mot.

Pour interpréter, le thérapeute fait usage de son propre inconscient, de son empathie, de ses intuitions ainsi que de ses référents théoriques (Greenson, 1977, 55). Dans la situation plurilingue, ces ressources ne sont pas suffisantes à la compréhension du patient ; l'interprète devient nécessaire et l'information qui parvient au thérapeute est en partie interprétée. 
Certains auteurs psychodynamiques relèvent le double sens du mot interprétation dans le contexte de la thérapie plurilingue (Darling, 2004, 258; Kouassi, 2001, 139; M’Barga, 1983, 296). L'interprète est à la fois quelqu'un qui peut traduire une signification d'une langue à une autre et quelqu'un qui peut saisir des significations latentes ou donner un sens à la communication inconsciente et la mettre en mots. Il permet d'aller du sens apparent, manifeste et littéral au sens latent, profond et caché (Darling, 2004, 258; Kouassi, 2001, 139; M'Barga, 1983, 296). Il permet de comprendre une dimension cachée de la communication, constituée, par exemple, de métaphores. L'ethnopsychiatrie française propose même d'utiliser les difficultés de traduction comme leviers thérapeutiques (De Pury, 1998, 125-126). Dans ces situations, l'interprète devient un informateur culturel qui explique les différents mots, et les significations associées qui permettent de décrire la pathologie, le comportement, les rituels. Son interprétation peut alors être considérée comme une forme d'intervention thérapeutique (Khelil, 2004, 6), car elle rend explicite des significations inconscientes. Il y a ainsi des similitudes entre le travail d'interprétation de l'interprète et celui du thérapeute: chacun traite un ensemble d'informations pour en dégager un sens et le partager d'une manière assimilable à son interlocuteur. En quelque sorte, tout comme l'interprète, le thérapeute doit trouver un équivalent dans une autre langue: il transmet au patient, dans une forme mentalisée, plus assimilable, le sens qui s'est dégagé de sa communication inconsciente.

Avant de discuter des implications de la présence d'un interprète dans la dynamique thérapeutique, il nous faut d'abord explorer le concept de neutralité. En effet, neutralité et interventions thérapeutiques sont intrinsèquement liées.

\section{La neutralité}

Tant dans l'interprétariat que dans la psychanalyse, la neutralité a représenté un idéal. De fait, Freud a initialement considéré les réactions contretransférentielles comme une entrave au processus thérapeutique. L'analyste devait rester neutre et impénétrable afin d'éviter de nuire à la liberté des associations et de donner libre cours au jeu de la projection (Dispaux, 2007, 670). Cette conception est notamment illustrée par la métaphore de l'écran blanc sur lequel la réalité du patient est projetée intacte. Aujourd'hui, des auteurs psychanalytiques avancent que le thérapeute est plutôt co-instigateur du transfert (Gabbard, 2000, 50-51), ou selon l'expression de Bot et Wadensjö $(2004,373)$, un participant actif d'un échange intersubjectif. Une évolution similaire est observée dans la posture de l'interprète. 


\section{Neutralité de l'interprète}

La question de la neutralité est centrale en interprétariat en soins de santé. Les auteurs qui se prononcent sur la neutralité des interprètes peuvent être sommairement divisés entre les tenants d'une posture neutre de l'interprète et ceux qui considèrent la neutralité comme plus ou moins impossible et qui soutiennent, comme corollaire à cette impossibilité une relation de collaboration entre praticien et interprète.

La neutralité fait partie des enjeux mis de l'avant dans la majorité des codes de déontologie et normes de pratique pour les interprètes communautaires dans le domaine de la santé, au même titre que la confidentialité et l'exactitude/exhaustivité (Bancroft, 2005, 19). On trouve des exemples de principes éthiques découlant de la neutralité dans des injonctions telles que ne donner aucun conseil, ne permettre aucune influence de ses sentiments ou de ses convictions sur le travail, et ne pas partager d'opinion, même si demandé. Cet accent sur la neutralité est un effet direct de la référence (implicite ou explicite) à l'interprétariat de conférence: dans ce contexte, l'interprétation est conçue comme ne devant en aucune manière interférer avec le discours.

Ceux qui préconisent la neutralité des interprètes dans le domaine de la santé mentale sont, pour la plupart, des psychiatres qui soulignent le risque d'erreurs de traduction, et les risques associés à la qualité des soins (Demetriou, 1991, 66-68; Farooq et Fear, 2003). Pour réduire ce risque, ils fournissent des directives strictes (Miletic et al., 2006) ou même, ils considèrent comme impossible d'offrir efficacement des soins psychologiques en présence d'un interprète (Yahyaoui, 1988). La neutralité sert ainsi à assurer la confiance du médecin, rendre la pratique de l'interprète transparente et guider les interprètes en toutes circonstances. Pour ces praticiens, l'interprète idéal est représenté par la métaphore de la machine à traduire. En revanche, ceux qui préconisent une approche collaborative voient l'interprète comme partie prenante de l'interaction. Ils proposent d'inclure les interprètes comme membres à part entière de l'équipe de soins (Bot, 2003; Bot, 2005; Raval, 2005; Raval et Maltby, 2005).

Suite à ces considérations concernant la neutralité du thérapeute et de l'interprète, deux questions paraissent importantes à poser: "la neutralité est-elle possible? » et « est-elle désirable?». Des études empiriques ont fourni des réponses à ces questions clés.

\section{La neutralité est-elle possible?}

Le travail pionnier dans la recherche en interprétariat communautaire de Kaufert et Koolage (1984), et plus tard celui de Wadensjö (1998), a claire- 
ment indiqué que les interprètes ne peuvent pas éviter d'influencer le dialogue. Ils jouent un double rôle en tant que traducteurs (relayant le message) et coordinateurs, dans le dialogue, des tours de parole. Six types de traductions ont été identifiés par Wadensjö (1998) : fermée, élargie, réduite, de substitution, de synthèse, ou aucune traduction. Parmi ceux-ci, la traduction fermée pourrait être considérée comme neutre puisqu'elle consiste en une traduction mot pour mot. Toutefois, même dans le cas où il est demandé à l'interprète de traduire tout ce qui a été dit de la façon dont cela a été dit, il est rarement possible de le faire. En réalité, la traduction mot-à-mot ne garantit pas la transmission adéquate du sens. L'utilisation d'autres types de traduction peut alors être considérée comme nécessaire plutôt que comme des erreurs tel que certains auteurs le présentent (Farooq et Fear, 2003; Flores et al., 2000 ; Karliner et al., 2007). D’un point de vue linguistique, les interprètes ne peuvent éviter d'insérer leurs propres connaissances ou perspectives dans l'interaction. Qui plus est, les patients ne considèrent pas les interprètes comme invisibles. En situation de psychothérapie, ces derniers rapportent même de fortes réactions émotionnelles envers les interprètes (Miller et al., 2005, 31). Tout comme il est impossible pour le thérapeute d'être totalement neutre, il est impossible pour l'interprète d'agir comme une non-personne (Wadensjö, 1998, 79).

\section{La neutralité est-elle désirable?}

Malgré cette impossibilité d'être totalement neutre, l'approximation de la neutralité, chez le thérapeute comme chez l'interprète, peut être utilisée pour viser des effets spécifiques, tels que le développement du transfert et l'émergence des représentations du patient (Greenson, 1977, 50; Wadensjö, 1998, 79). La neutralité consiste également à ne pas agir son contre-transfert et contenir ses impulsions en vue d'accompagner le patient dans sa propre compréhension de sa dynamique psychique. Aubert $(2008,71)$ souligne la ressemblance entre la demande à l'interprète de «tout traduire» et la règle fondamentale de l'association libre, qui invite le patient à dire tout ce qui lui traverse l'esprit, pour donner accès à ce qui constitue sa réalité psychique. Selon cet auteur, cette demande adressée à l'interprète aurait l'avantage de lui indiquer qu'il n'est pas mis en position de négocier avec le patient ce qu'il convient de transmettre ou pas au thérapeute, et que c'est le thérapeute qui conduit les entretiens.

S'effacer n'est cependant pas souhaitable dans tous les contextes. En effet, l'appartenance ethnoculturelle et sociale façonne le langage utilisé pour 
exprimer des états intérieurs, et elle configure le langage des émotions et des expériences subjectives. Il devient difficile de comprendre la réalité de l'autre sans accès à son contexte. L'interprète désirant ne pas sortir de sa neutralité risque de priver le thérapeute d'informations essentielles à la compréhension, auxquelles lui-même a peut-être accès.

Par exemple, les mots ont une dénotation et une connotation. La dénotation est le sens spécifique du mot, tandis que la connotation réfere à la signification associée ou à la coloration émotionnelle de la parole (Westermeyer et Janca, 1997, 298). La connotation peut facilement se perdre si l'interprète traduit mot pour mot ou ne possède pas les connaissances ou l'expérience de l'univers ethnoculturel et social des interlocuteurs. Inversement, un interprète qualifié sera à même de trouver un sens équivalent ou de contextualiser et expliquer la signification de mots spécifiques.

Il n'est pas surprenant que les auteurs d'approche psychanalytique insistent sur l'implication subjective de l'interprète dans le processus thérapeutique. L'interprète doit être conscient de ses propres préjugés et être engagé dans le travail thérapeutique, en particulier la réflexion sur sa propre parole (Khelil, 2004, 6). Pour Kouassi (2001, 141), l'interprète ne doit pas seulement s'effacer derrière l'acte de traduction, il devrait laisser apparaître un certain degré de sa propre subjectivité. Se faisant, il peut servir de modèle au patient et ainsi l'aider à établir des liens entre l'intérieur et l'extérieur, le passé et le présent (en admettant que l'interprète ait intégré harmonieusement ce que le patient n'arrive pas encore à faire).

L'utilisation de la première personne du singulier (le «je») pour transmettre les paroles du patient est un moyen recommandé pour témoigner de la subjectivité du patient (Rechtman, 1992, 351-355 ; 1997, 362). Rechtman donne l'exemple qui suit. Pour la traduction de «Il pense qu'il a vu un fantôme», le patient peut avoir dit «J'ai vu un fantôme» ou «Je pense avoir vu un fantôme». Le sens de ces deux phrases n'est évidemment pas le même: un doute est inclus dans la deuxième et laisse entendre une certaine distance par rapport à l'expérience rapportée. L'utilisation de la première personne apparaît plus facile, plus rapide à traduire et présente l'avantage de restaurer l'expression de la position subjective du patient. En s'effaçant derrière le «je», ajoute l'auteur, l'interprète peut alors faciliter l'émergence de la dynamique transférentielle patient-thérapeute. Cette tâche est toutefois difficile et peut être ressentie par l'interprète comme une dissonance entre l'idéal de neutralité imposé par les codes déontologiques et l'implication émotionnelle demandée par le thérapeute et les situations thérapeutiques (Goguikian 
Ratcliff et Suardi, 2006, 46). De plus, participer à l'expérience et aux mots du thérapeute et du patient peut affaiblir l'identité personnelle de l'interprète (Bradford et Munoz, 1993, 58), en particulier si celui-ci a vécu une histoire migratoire similaire à celle du patient (Rechtman, 1992, 351-353). L'utilisation de la troisième personne peut alors introduire une distance émotionnelle, parfois nécessaire.

Réifier le thérapeute en un écran blanc et l'interprète en une machine à traduire suggère une conception épistémologique objectivante, autrement dit une croyance en l'existence d'une réalité objective, extérieure à l'observateur, qui peut être «découverte». Cette conception de la réalité suppose, pour un thérapeute détaché, d'éviter toute idée préconçue afin de découvrir la vérité objective du patient (Gabbard, 2000, 49). Des perspectives théoriques contemporaines telles que le co-constructivisme et l'intersubjectivité mettent au défi cette conception en adoptant le dialogisme qui reconnaît l'influence de chaque participant sur les autres participants (Gabbard, 2000, 50-51).

\section{Considérations selon les canaux de communication}

Dans le contexte d'une rencontre à trois, la présence de l'interprète modifie chacun des trois canaux de communication : le verbal, le non-verbal et le projectif (transfert et contre-transfert).

\section{Canal verbal}

Les praticiens tant de la médecine somatique que de la santé mentale s'entendent pour reconnaître l'importance de l'interprète communautaire dans l'établissement de la communication verbale, ou en d'autres termes dans sa posture d'agent linguistique selon l'expression de Leanza (2005, 186-187). Les études en linguistique montrent que, malgré les apparences, cette posture d'agent linguistique est des plus complexes. Comme il est présent dans l'interaction, l'interprète doit faire des choix dans l'immédiateté concernant la forme (les mots et la grammaire) de l'information à transmettre. Ces choix le rendent encore plus présent dans l'interaction (Bot et Wadensjö, 2004, 360). En effet, il n'est pas rare qu'une gamme de mots ou d'expressions puissent traduire un seul mot. Ceux-ci ont des connotations et des sens différents selon les contextes et la personne qui les emploie. Les règles de grammaire changeant d'une langue à l'autre, l'interprète est amené à faire des choix qui transformeront nécessairement le discours. 


\section{Canal non verbal}

Aussi bien le corps du patient que son discours sont source d'une multitude d'indices non-verbaux, tels que le regard, l'intonation, le rythme ou les accents. Toute situation interlinguistique et interculturelle rend encore plus complexe le décodage de ces informations transmises par le canal non-verbal (Westermeyer, 1993, 129). Bien que le thérapeute puisse observer les attitudes corporelles, il peut lui être difficile de les relier aux mots, puisque les deux informations lui parviennent de façon décalée dans le temps (Haenel, 1997, 68). En commentant la situation ou en répondant aux questions du thérapeute, l'interprète permet alors l'articulation entre le verbal et le nonverbal. L'interprète peut être en mesure de fournir des informations sur certains comportements non-verbaux (p. ex. l'habillement ou le contact visuel) dans la situation où le thérapeute n'en saisit pas le sens. Se faisant, il sort de sa posture d'agent linguistique pour en adopter une autre, celle d'agent du monde vécu (ou agent communautaire, Leanza, 2005 186-187). Certains auteurs nous mettent toutefois en garde: les codes culturels sont difficiles à résumer et à communiquer (p. ex. Piret, 1991, 4). En outre, la possibilité de recevoir ce type d'information dépend du contrat, si contrat il y a, liant l'interprète et le thérapeute, c'est-à-dire des rôles que l'interprète peut prendre pendant, mais aussi avant et après, la rencontre.

On trouve des indices non-verbaux dans la langue elle-même, et ceux-ci nous renseignent sur le statut mental du patient (Jackson et al., 2008, 141142; Westermeyer et Janca, 1997, 305 ; Westermeyer, 1993, 129). L'interprète peut donner accès à de nombreuses informations non-verbales essentielles à l'évaluation, telles que la vitesse d'élocution, la cohérence et la répétition.

Dans une perspective psychanalytique, la langue est plus qu'un moyen de transmission des représentations et des affects. Elle est en soi un matériel à interpréter dans le processus thérapeutique. Chaque langue possède des connotations différentes (M’Barga, 1983, 296). Celles liées à la langue maternelle peuvent inclure notamment le désir et la régression, et celles en lien avec la langue du thérapeute peuvent inclure le pouvoir et l'histoire du passé colonial. Piret $(1991,9)$ mentionne le désir du patient migrant de se réconcilier avec sa langue maternelle, mais aussi de s'en libérer, celle-ci pouvant représenter les premiers attachements aliénants. Le choix par le patient de la langue dans laquelle il s'exprime n'est donc pas neutre ou seulement pragmatique. Il s'agit d'une dimension supplémentaire en psychothérapie qui doit être soigneusement évaluée et interprétée. 


\section{Contre-transfert}

Le contre-transfert est le canal de communication spécifique à l'approche psychanalytique, et c'est celui qui est le plus discuté dans la littérature traitant de l'interprétariat en thérapie analytique. La présence de l'interprète dans la rencontre thérapeutique modifie de fait la dynamique relationnelle, et implique des réactions émotionnelles complexes (Miller et al., 2005, 28-37). Chacun des trois protagonistes répondra affectivement, consciemment ou non, aux deux autres personnes.

La rencontre à trois implique pour le thérapeute, mais également pour l'interprète, d'occuper une place dans le transfert du patient (Piret, 1991, 4). Le transfert du patient envers l'interprète devient d'un grand intérêt puisque l'alliance thérapeutique se crée souvent en premier, et parfois même principalement, avec lui (Miller et al., 2005, 30-31; Raval et Smith, 2003, 13; Westermeyer, 1993, 129). Différents exemples sont mentionnés dans la littérature. Le patient peut voir l'interprète comme, par exemple, un compatriote ayant subi les mêmes difficultés, le considérant alors comme un allié (Aubert, 2008, 70-71; Valero-Garces, 2005, 1-3). À l'inverse, ce dernier peut représenter une menace du fait d'une possibilité de violation de confidentialité (Bolton, 2002, 107; Bot et Wadensjö, 2004, 373-375) et de la stigmatisation dans la communauté qui pourrait en découler. Le patient pourrait également ressentir un sentiment de honte dans le fait de se dévoiler à un compatriote. L'existence de deux canaux de transfert, c'est-à-dire l'interprète et le thérapeute, rend davantage visibles les clivages du patient. L'interprète peut être investi comme le mauvais objet s'il est perçu, par exemple, comme quelqu'un pouvant dénoncer les opinions politiques du patient; et le thérapeute, en tant que bon objet tel un sauveur puissant et protecteur (Aubert, 2008, 70-71; Bot et Wadenjö, 2004, 369-371; Haenel, 1997, 69-71). Bien entendu, interprète et praticien peuvent tour à tour être associés aux mauvais ou aux bons objets (Aubert, 2008, 70-71; Haenel, 1997, 69-71).

L'interprète n'est pas qu'un objet de transfert. Il ne peut échapper à ses réactions contre-transférentielles envers le patient, qu'il en ait conscience ou non (Piret, 1991, 4). Le contre-transfert des interprètes peut par exemple s'exprimer par un désir de surprotéger le patient (Haenel, 1997, 70), de normaliser le discours du patient pour éviter la honte que suscite la pathologie (Westermeyer, 1993, 130), ou par un sentiment de menace lié à une réalité objective (si interprète et patient appartiennent à des ethnies en conflit, par exemple) ou fantasmée. Les thérapeutes peuvent tirer profit des moments où interprète et patient communiquent dans la langue du patient afin d'en 
observer la dynamique relationnelle (Rechtman, 1992, 355; Westermeyer 1993, 130). Cela permet d'évaluer l'effet du discours du patient sur l'interprète, ainsi que la charge émotionnelle qui accompagne les propos. Ceci est d'autant plus utile que l'émotion peut être difficile à traduire (Khelil, 2004, 3).

Des alliances peuvent se créer entre deux des protagonistes aux dépens du troisième (Bradford et Munoz, 1993, 58-59) : le patient peut s'allier à l'interprète ou au thérapeute; et l'interprète, au patient ou au thérapeute. Elles peuvent, par exemple, représenter des identifications à la victime ou à l'agresseur. En outre, l'interprète peut présenter des sentiments d'admiration envers le thérapeute, tout en dépréciant le patient (Haenel, 1997, 70).

De forts sentiments d'exclusion, d'impuissance et de perte de contrôle chez les thérapeutes sont fréquents dans les consultations avec interprètes (Bradford et Munoz, 1993, 58; Miller et al., 2005, 33 ; Raval, 1996, 34 ; Raval et Smith, 2003, 21-24); dans certains cas, ceux-ci convergeraient avec les sentiments du patient. L'identification projective peut aussi prendre la forme d'une immobilisation des mouvements de pensée du thérapeute (Aubert, 2008,72 ). Ceci se produit «dans un contexte où le thérapeute est déstabilisé, interpellé dans son rapport au monde, au même titre que le patient » (Aubert, 2008, 72).

En fait, ces différents processus psychiques intrinsèques à la relation représentent une opportunité de compréhension de la dynamique des patients, et ne pas les considérer nuirait au processus thérapeutique. À l'occasion, la communication à un niveau affectif peut être bloquée si l'interprète est submergé par ce qui est dit. Une telle difficulté, lorsqu'analysée, permet toutefois une compréhension plus fine des difficultés du patient (Darling, 2004, 261). Le contre-transfert est donc «à la fois un obstacle et un outil essentiel au travail thérapeutique» (Normandin et Ensink, 2007, 58), non seulement chez le thérapeute, mais également pour l'interprète.

\section{Résistances}

Non seulement l'analyse porte-t-elle sur le matériel produit par le biais des réactions transférentielles, mais également sur les résistances. Ce concept psychanalytique permet d'éclairer certaines particularités de la dynamique du patient-thérapeute-interprète. En effet, l'interprète est le seul à avoir accès aux lapsus, aux flous, aux arrêts, aux doubles sens, aux hésitations, etc., dans le discours. L'écoute analytique doit ainsi se situer autant du côté du thérapeute que de l'interprète (Piret, 1991, 5), ce qui n'est pas sans poser la 
question de la formation des interprètes. À ce titre, il est important que l'interprète rapporte les formes de résistances du patient (Piret, 1991, 5-6). Pour ne donner que quelques exemples de modes de résistance retrouvés dans la situation thérapeutique plurilingue, le patient peut se réfugier dans le flou de la traduction plutôt que d'assumer ce qu'il a dit, comme ses propres lapsus. Il peut aussi ne s'adresser qu'à l'interprète afin de se protéger et d'éviter de s'engager pleinement dans le processus thérapeutique. Encore, il peut utiliser un registre technique ou le jargon médical pour se distancer de ses affects. Il peut changer de langue (code-switching) pour éviter l'intensité des affects dans la première langue (Oquendo, 1996, 614-617). Cette tactique serait rendue possible du fait de l'indépendance des langues, du manque de chevauchement entre les mémoires et les associations entre les deux langues. Le code-switching n'est pas le propre du patient. Le thérapeute peut également y avoir recours en fonction de l'effet souhaité: utiliser la langue maternelle afin de surmonter la résistance, ou utiliser la langue seconde afin de diminuer l'intensité émotionnelle (Oquendo, 1996, 614-617).

\section{Intégrer l'interprète au cadre}

Afin de profiter des connaissances que l'interprète a acquises sur le patient, un temps de réflexion avec l'interprète après la séance est également préconisé (Darling, 2004, 266; Rechtman, 1992, 355; Rechtman et Welsh, 1993, 155). Les réactions contre-transférentielles de l'interprète peuvent alors être explorées, tout comme la qualité du discours du patient et le matériel culturel. Un tel débriefing peut s'avérer particulièrement utile suite à un agir contre-transférentiel du thérapeute ou de l'interprète, et dans la situation où la réflexion et l'action appropriées n'étaient pas possibles durant la séance même (Darling, 2004, 266). Qui plus est, une réflexion partagée a l'avantage de faciliter le développement de l'alliance de travail dans le temps. En effet, ce n'est pas la clarté des consignes données à l'interprète qui explique le mieux l'ajustement interprète-thérapeute, mais bien la continuité dans le temps de l'alliance de travail (Goguikian Ratcliff et Suardi, 2006, 45). Elle permet de créer des représentations communes et par le fait même, d'intégrer le travail de l'interprète au cadre thérapeutique.

\section{Conclusion}

Interpréter est le cœur de l'activité des interprètes communautaire et des thérapeutes analytiques. La démarche de ces premiers vise à dégager le sens dans une langue et le transmettre au patient ou au thérapeute dans une autre, 
alors que celle des thérapeutes, à traduire le langage de l'inconscient en un langage conscient, mentalisé.

Interpréter, et non traduire, nécessite de considérer que la neutralité prend sens différemment selon les contextes. Sokol (2010, 18-19), dans une réflexion récente sur cette question, démontre que «la neutralité est un concept vague et abstrait, d'une grande ambiguïté si l'on est amené à le questionner. [Il s'agit d'] un concept générique qui en cache en réalité plusieurs autres : transparence, recul, impartialité » (Sokol, 2010, 61). Ce constat invite à une réflexion-négociation, entre le thérapeute et l'interprète, à propos de la place à accorder à la neutralité et à leur propre subjectivité dans la communication afin d'en tirer profit selon les contextes.

Bien que le thérapeute soit la personne responsable de la séance thérapeutique, cet article suggère que le rôle de l'interprète ne s'arrête pas à celui d'agent linguistique (canal verbal) dans la thérapie analytique. Il a de facto un pouvoir qui va au-delà et qui influe sur la dynamique relationnelle (Leanza et Boivin, 2008, 4-10; Leanza et al., 2010, 1893-1895). Il peut entre autres débrouiller la communication tant au niveau non verbal que contre-transférentiel. Pour ce faire, une formation appropriée, une pratique réflexive partagée, ainsi que la continuité dans le temps de l'alliance de travail, semblent essentielles.

Le présent article propose également des pistes de réflexion sur la façon d'intégrer l'interprète à la rencontre thérapeutique tant dans sa position d'interlocuteur que de passeur, de manière à bénéficier de ses connaissances au niveau linguistique, mais aussi de ses connaissances en lien avec sa position intermédiaire entre deux personnes et entre deux univers linguistiques et socioculturels, pour in fine, peut-être, le constituer cothérapeute dans la thérapie plurilingue.

Isabelle Boivin, Université de Sherbrooke

Camille Brisset, Université Laval

Yvan Leanza, Université Laval

\section{Références}

AUBERT, A.-E., 2008, La transformation du côté du thérapeute comme préalable au changement du patient en situation transculturelle, Pratiques psychologiques, 14, 67-78.

BANCROFT, M., 2005, The Interpreter's World Tour: An Environmental Scan of Standards of Practice for Interpreters, Ellicott City, Maryland, National Council on Interpreting in Health Care. 
BLEGER, J., 1979, Psychanalyse du cadre psychanalytique, in Kaës, R., Missenard, A., Kaspi, R., Anzieu, D., éds, Crise, rupture et dépassement, Dunod, Paris, 255-285.

BOLTON, J., 2002, The third presence : a psychiatrist experience of working with non-English speaking patients and interpreters, Transcultural Psychiatry, 39, 97-114.

BOT, H., 2003, The myth of the uninvolved interpreter interpreting in mental health and the development of a three-person psychology, in Brunette, L., Bastin, G., Hemlin, I., Clarke, H., éds, The Critical Link 3, Amsterdam, John Benjamins Publishing company, 27-35.

BOT, H., 2005, Dialogue Interpreting in Mental Health, Rodopi, Amsterdam.

BOT, H., WADENSJÖ, C., 2004, The presence of a third party: a dialogical view on interpreter-assisted treatment, in Wilson, J., Drozdek, B., éds, Broken Spirits, The Treatment of Traumatized Asylum Seekers, Refugees, War and Torture Victims, New York, BrunnerRoutledge, 355-378.

BRADFORD, D. T., MUNOZ, A., 1993, Translation in bilingual psychotherapy, Professional Psychology: Research and Practice, 24, 52-61.

DARLING, L., 2004, Psychoanalytically-informed work with interpreters, Psychoanalytic Psychotherapy, 18, 255-267.

DE PURY, S., 1998, Traité du malentendu. Théorie et pratique de la médiation interculturelle en situation clinique, Le Plessis-Robinson, Institut Synthélabo.

DEMETRIOU, S., 1991, Interpreters and psychiatry, in Minas, I. H., éd., Cultural Diversity and Mental Health, Melbourne, Royal Australian and New Zealand College of Psychiatrists and Victorian Transcultural Psychiatry Unit, 65-69.

FAROOQ, S., FEAR, C., 2003, Working through interpreters, Advances in Psychiatric Treatment, 9, 104-109.

FLORES, G., ABREU, M., SCHWARTZ, I., HILL, M., 2000, The importance of language and culture in pediatric care: case studies from the Latino community, Journal of Pediatrics, $137,842-848$.

GABBARD, G. O., 2000, Psychodynamic Psychiatry in Clinical Practice, American Psychiatric Press, Washington, DC.

GOGUIKIAN RATCLIFF, B., CHANGKAKOTI, N., 2004, Le rôle de l'interprète dans la construction de l'interculturalité dans un entretien ethnopsychiatrique, L'Autre, 5, 255264.

GOGUIKIAN RATCLIFF, B., SUARDI, F., 2006, L'interprète dans une consultation thérapeutique: Conceptions de son rôle et difficultés éprouvées, Psychothérapies, 26, 37-49.

GREENSON, R. R., 1968, Technique et pratique de la psychanalyse, Presses universitaires de France, Paris.

GREENSON, R. R., 1977, Technique et pratique de la psychanalyse, Presses universitaires de France, Paris.

HAENEL, F., 1997, Aspects and problems associated with the use of interpreters in psychotherapy of victims of torture, Torture, 7, 68-71.

JACKSON, C., ZATZICK, D., HARRIS, R., GARDINER, L., 2008, Loss in translation. Considering the critical role of interpreters and language in psychiatric evaluation of non-English-speaking patients, in Loue, S., Sajatovic, M., éds., Diversity Issues in the Diagnosis Treatment and Research of Mood Disorders, Oxford, New-York, 135-163.

KARLINER, L. S., JACOBS, E. A., CHEN, A. H., MUTHA, S., 2007, Do professional interpreters improve clinical care for patients with limited English proficiency? A systematic review of the literature, Health Services Research, 42, 727-754.

KHELIL, K., 2004, Des impasses de la traduction aux ouvertures de la psychothérapie, Parole Sans Frontière, http://www.p-s-f.com/psf/spip.php?article32\&var_recherche=Khelil, consulté le 31 mars 2009. 
KOUASSI, K., 2001, Approche psychothérapeutique en clinique transculturelle. Triade thérapeute/patient/interprète, Champ psychosomatique, 23, 137-143.

LEANZA, Y., 2005, Roles of community interpreters in pediatrics as seen by interpreters, physicians and researchers, Interpreting, 7, 167-192.

LEANZA, Y., BOIVIN, I., 2008, Interpréter n'est pas traduire. Enjeux de pouvoir autour de l'interprétariat communautaire, actes en ligne du colloque international L'éducation en contextes pluriculturels: la recherche entre bilan et prospectives, Université de Genève, Genève.

LEANZA, Y., BOIVIN, I., ROSENBERG, E., 2010, Interruptions and resistance : a comparison of medical consultations with trained and family interpreters, Social Science and Medicine, 70, 1888-1895.

M'BARGA, J.-P., 1983, Relation thérapeutique transculturelle: Interprétariat, interprétation, Études psychothérapeutiques, 54, 292-300.

MILETIC, T., PIU, M., MINAS, H., STANKOVSKA, M., STOLK, Y., KLIMIDIS, S., 2006, Guidelines for Working Effectively with Interpreters in Mental Health Settings, http://www.vtpu.org.au/docs/interpreter/VTPU_GuidelinesBooklet.pdf, consulté le 15 juin 2008.

MILLER, K. E., MARTELL, Z. L., PAZDIREK, L., CARUTH, M., LOPEZ, D., 2005, The role of interpreters in psychotherapy with refugees: An exploratory study, The American Journal of Orthopsychiatry, 75, 27-39.

NORMANDIN, L., ENSINK, K., 2007, La GAC: Grille d'analyse du contre-transfert dans le traitement des troubles graves de la personnalité, Santé mentale au Québec, 32, 1, 57-74.

OQUENDO, M. A., 1996, Psychiatric evaluation and psychotherapy in the patient's second language, Psychiatric Services, 47, 614-618.

PIRET, B., 1991, La psychothérapie avec interprète est-elle possible? Parole sans frontière, http://www.p-s-f.com/psf/spip.php?article23\&var_recherche=Piret, consulté le 31 mars 2009.

PÖCHHACKER, F., 1999, Getting organized: the evolution of community interpreting, Interpreting, 4, 125-140.

RAVAL, H., 1996, A systemic perspective on working with interpreters, Clinical Child Psychology and Psychiatry, 1, 29-43.

RAVAL, H., 2005, Being heard and understood in the context of seeking asylum and refuge: Communicating with the help of bilingual co-workers, Clinical Child Psychology and Psychiatry, 10, 197-216.

RAVAL, H., MALTBY, M., 2005, Not getting lost in translation : establishing a working alliance with co-workers and interpreters, in Flaskas, C., Mason, B., Perlesz, A., éds., The Space between Experience, Context, and Process in the Therapeutic Relationship, Karnac Books, London, 63-78.

RAVAL, H., SMITH, J., 2003, Therapist'experiences of working with language interpreters, International Journal of Mental Health, 32, 6-31.

RECHTMAN, R., 1997, Transcultural psychotherapy with Cambodian refugees in Paris, Transcultural Psychiatry, 34, 359-375.

RECHTMAN, R., 1992, L'intraduisible culturel en psychiatrie, L'Évolution psychiatrique, 57, 347-365.

RECHTMAN, R., WELSH, G., 1993, Approche transculturelle des patients non francophones originaires du Sud-est asiatique dans le dispositif psychiatrique du XIII ${ }^{e}$ arrondissement de Paris, Santé mentale au Québec, 1, 143-162.

SOKOL, A., 2010, L'interprète en entretien clinique: Quel rôle pour quelle neutralité?, Université de Lausanne, Lausanne. 
VALERO-GARCÉS, C., 2005, Emotional and psychological effects on interpreters in public services: A critical factor to bear in mind, Translation Journal, 9, from http://www. accurapid.com/journal/33ips.htm, consulté le 29 février 2009.

WADENSJÖ, C., 2002, The double role of a dialogue interpreter, in Pöchhacker, F., Shlesinger, M., éds., The Interpreting Studies Reader, Routledge, London, 354-370.

WADENSJÖ, C., 1998, Interpreting as Interaction, Longman, Londres.

WESTERMEYER, J., 1993, Cross-cultural psychiatric assessment, in Gaw, C., éd, Culture, Ethnicity and Mental Illness, American Psychiatric Press, Washington, DC, 125-144.

WESTERMEYER, J., JANCA, A., 1997, Language, culture and psychopathology: Conceptual and methodological issues, Transcultural Psychiatry, 34, 291-311.

YAHYAOUI, A., 1988, Consultation familiale ethnopsychanalytique: le cadre interculturel, in Yahyaoui, A., éd., Troubles du langage et de la filiation chez le Maghrébin de deuxième génération, La pensée sauvage, Grenoble, 48-67. 